\section{P02 UNHEALTHY PARTNER RELATIONSHIPS MAY INDIRECTLY CONTRIBUTE TO EARLY CHILDHOOD OBESITY}

${ }^{1} J u l i e$ Kapp*, ${ }^{2}$ Allison Kemner, ${ }^{3}$ Dana Duren. ${ }^{1}$ Health Management and Informatics, University of Missouri School of Medicine, Columbia, US; ${ }^{2}$ Parents as Teachers National Center, Saint Louis, US: ${ }^{3}$ Orthopedic Surgery; Pathology and Anatomical Sciences, University of Missouri School of Medicine, Columbia, US

\subsection{6/jech-2021-SSMabstracts.92}

Background Curtailed sleep (shorter than average sleep duration) in a child's first 1,000 days of life is associated with early childhood obesity. Children sharing a room with a parent, compared to independent sleepers, have less nighttime sleep, shorter sleep stretches, and unsafe sleep practices. The goal is to optimize children's sleep duration and consolidation. Empirical examination of children's sleep behaviors in realworld, population-based samples is limited. From a diverse sample of U.S. families, we deconstructed the mechanisms for bedsharing behaviors. We hypothesize that unhealthy relationships with family members and partners, and nurturing behaviors, are associated with bedsharing.

Methods This analysis included 4,539 U.S. mother-child dyads actively enrolled in the Parents as Teachers (PAT) home visiting program July 2015 through July 2020 from affiliate sites meeting the highest programmatic quality standards. Exclusion criteria due to potential confounding consisted of: substance abuse, child abuse, incarceration, unstable housing, and mental illness, or missing data on the outcome variable. In a multivariable logistic regression model examining the outcome of bedsharing 'sometimes' or 'always' compared to 'never,' we investigated maternal relationships with family, partners, and maternal nurturing as scored lower than the target on the Life Skills Progression instrument, while controlling for low income, low education, young parent $(<$ age 21), multiple children younger than age six, single parent, first time parent, child's age, and number of home visits.

Results In our sample of children ages 0 to 4 years, $44.8 \%$ bedshare. Our sample included: $10.5 \%$ young parents, $25.7 \%$ multiple children younger than six, $19.4 \%$ single parents; $22.8 \%$ first time parents, $35.5 \%$ low education, $71.7 \%$ low income, and $10.4 \%, 14.9 \%$, and $6.3 \%$ respectively scoring less than target on the quality of the family and partner relationships, and nurturing scales. Mothers scoring low on the quality of the partner relationship are more likely to bedshare (aOR: 1.3 (95\% CI: 1.11.6)); quality of the family relationship, and nurturing scores were not statistically significant (model c-statistic: 0.59).

Conclusion Women with multiple partners, violent relationships, who have lost contact with partners, have frequent verbal fights with partners, or who have inconsistent or conditional partner support are more likely to bedshare. Addressing these social determinants of health should be a priority before addressing parenting behaviors.

\section{\begin{tabular}{|l|l}
\hline P03 DECONSTRUCTING RISK FACTORS FOR EARLY \\
\hline
\end{tabular} CHILDHOOD OBESITY IN A POPULATION-BASED SAMPLE}

${ }^{1} J u l i e$ Kapp*, ${ }^{2}$ Allison Kemner, ${ }^{3}$ Dana Duren. ${ }^{1}$ Health Management and Informatics, University of Missouri School of Medicine, Columbia, USA; ${ }^{2}$ Parents as Teachers National Center, Saint Louis, USA; ${ }^{3}$ Orthopedic Surgery; Pathology and Anatomical Sciences, University of Missouri School of Medicine, Columbia, USA

10.1136/jech-2021-SSMabstracts.93
Background A responsive parenting intervention was found to mitigate behaviors that contribute to early childhood obesity: after intervention, mothers were less likely to pressure infants to finish a bottle, use food to soothe, or put their baby to bed with a bottle. However, whether these findings translate to real-world home environments is uncertain given the intervention sample was largely White and well-educated, with incomes greater than $\$ 50,000$. We hypothesize there are mechanistic barriers to responsive parenting behaviors among diverse U.S. families; specifically, low maternal nurturing, low discipline, and low support of child development.

Methods This analysis included 7,407 U.S. mother-child dyads enrolled in the Parents as Teachers (PAT) home visiting program July 2015 through July 2020 from affiliate sites meeting the highest programmatic quality standards. Exclusion criteria due to avoid potential confounding consisted of: substance abuse, domestic violence, child abuse, incarceration, unstable housing, and mental illness, or missing data outcome variable data. In a multivariable logistic regression model examining the outcome of putting the baby to bed with a bottle 'sometimes' or 'always' compared to 'never,' we investigated maternal nurturing, discipline, and support of development as scored lower than the target on the Life Skills Progression instrument, while controlling for low income, low education, young parent ( $<$ age 21), multiple children younger than six years of age, single parent, first time parent, child's age, and number of home visits.

Results In our sample of children ages 0 to 4 years, $19.5 \%$ have been put to bed with a bottle. Our sample included: 9.0\% young parents, $26.1 \%$ multiple children younger than six, $17.3 \%$ single parents; $21.8 \%$ first time parents, $32.4 \%$ low education, $69.6 \%$ low income, and 5.9\%, 11.9\%, and $19.0 \%$ respectively scoring less than target on nurturing, discipline, and support of development. Mothers scoring low on support of development compared to scoring in the target range were more likely to put the baby to bed with a bottle (aOR: 1.3 (95\% CI: 1.1-1.6)) sometimes or always versus never; nurturing and discipline scores were not statistically significant (model c-statistic: 0.66).

Conclusion Maternal gaps in knowledge of child development, unrealistic expectations, or a passive parental role are independently associated with bottle use behaviors related to early childhood obesity. Home visiting programs such as PAT that reach a diverse population, are scaled nationally, and address these values and behaviors also have the potential to help mitigate early childhood obesity.

\section{P04 ASSOCIATION BETWEEN BREASTFEEDING DURATION AND COGNITIVE DEVELOPMENT UP TO AGE 14 AMONG CHILDREN FROM THE UK MILLENNIUM COHORT STUDY}

Reneé Pereyra-Elías*, Maria A Quigley, Claire Carson. National Perinatal Epidemiology Unit, Nuffield Department of Population Hea, University of Oxford, Oxford, UK

\subsection{6/jech-2021-SSMabstracts.94}

Background Duration of breastfeeding is associated with improved cognitive development, but it is unclear whether this relationship is real or due to confounding. This study evaluates how much of the observed association is explained by confounding of socioeconomic position (SEP) and maternal cognitive ability. 